\title{
Förderung der mündlichen Kommunikationsfähigkeit - Anforderungen an die schulische Praxis und die universitäre fachdidaktische Ausbildung von Russischlehrkräften
}

\author{
Heike Wapenhans (Berlin)
}

Общепризнанной целью изучения иностранных языков (ИЯ) явАяется практическое владение языком и его использование в качестве средства общения, в первую очередь, в устной речи. Это нашло своё отражение в документах образовательного характера, как на европейском, так и на общегерманском уровне и, конечно же, в учебных планах федеральных земель. Во всех этих документах подчёркивается особая значимость формирования устных форм коммуникативной компетенции. В статье обращается внимание на то обстоятельство, что Аля Аостижения этой цели требуется немало времени и усилий как обучаемых, так и обучающих. Последние призваны учесть конкретные условия и требования к учебной Аеятельности, чтобы целенаправленно способствовать развитию нужных навыков и умений учащихся. Поэтому необходима соответствующая подготовка будущих учителей ИЯ, вкАючающая приобретение и развитие профессиональных знаний и умений относительно выбора и применения подходящих учебных ситуаций, методов и приёмов, способов Аифференциации и оценки. Именно овладению определённым набором эффективных методов и приёмов, релевантных для обучения устной речи, стоит уделять больше внимания.

Anka Bergmann, Olga Caspers \& Wolfgang Stadler (Hg.)

Didaktik der slawischen Sprachen - Beiträge zum 1. Arbeitskreis in Berlin (12.-14.9.2016)

(C) 2018 innsbruck university press, ISBN 978-3-903187-11-5, DOI 10.15203/3187-11-5 


\section{Mündliche Kommunikationsfähigkeit als Ziel des Fremdsprachenerwerbs}

Das vorrangige Anliegen bzw. Ziel derer, die eine andere Sprache lernen, ist seit eh und je die mündliche Sprachbeherrschung. Fremdsprachenlernende wollen die gelernte bzw. zu lernende - gesprochene - Sprache verstehen und auch selbst sprechen. Schließlich dominiert auch in der Alltagskommunikation die gesprochene Sprache: Das Verhältnis von gesprochener zu geschriebener Sprache beträgt ca. 95\% zu 5\% (vgl. Wolff 2000: 11).

Die Realisierung dieses Ziels wird von Fremdsprachenlernenden allerdings als schwierig eingestuft. Lernende empfinden den Lernprozess in diesem Bereich als langwierig und anspruchsvoll, ihre Lernergebnisse bleiben oft hinter ihren Erwartungen zurück. Aus diesem Grund stellt die Mündlichkeit auch für Lehrende eine besondere Herausforderung dar: Sie sollen optimale Bedingungen, Szenarien und Gelegenheiten zur Entwicklung der mündlichen Fertigkeiten schaffen, die für mündliches kommunikatives Handeln und Diskursfähigkeit notwendig sind.

Die Schwierigkeiten im Umgang mit Mündlichkeit im Fremdsprachenerwerb erklären sich aus der Komplexität der einzelnen Komponenten und der sie konstituierenden Prozesse. Das Hör-/Hörsehverstehen als rezeptive Komponente wie auch das Sprechen als produktive Komponente sind für sich genommen höchst komplexe Prozesse, die mehrere Teilprozesse und -fertigkeiten umfassen und weitere Kompetenz- und Wissensbereiche, wie spezielles Sprachwissen (phonetisches, lexikalisches, grammatikalisches, pragmatisches, soziolinguistisches) sowie Sach- bzw. Weltwissen, aber auch verschiedene Strategien (z. B. Problemlöse-, Kompensations-, Vermeidungsstrategien) voraussetzen (vgl. Behr \& Wapenhans 2014: 134-137). Darüber hinaus verlangen die vielfältigen Erscheinungsformen oder Ausprägungen von medialer und konzeptioneller Mündlichkeit einen besonderen Zugang. So lässt sich Mündlichkeit inzwischen nicht mehr auf phonisch, flüchtig und face-to-face realisierte Sprache beschränken, sondern schließt phonisch/geschriebene und graphisch/gesprochene Mündlichkeit mit ein (vgl. Kurtz 2014: 118-119), auf die im Beitrag jedoch nicht weiter eingegangen wird. Das Primat der Mündlichkeit wird - ohne die Bedeutung der Schriftlichkeit geringzuschätzen - damit noch verstärkt und erklärt

Anka Bergmann, Olga Caspers \& Wolfgang Stadler (Hg.)

Didaktik der slawischen Sprachen - Beiträge zum 1. Arbeitskreis in Berlin (12.-14.9.2016)

(C) 2018 innsbruck university press, ISBN 978-3-903187-11-5, DOI 10.15203/3187-11-5 
die Notwendigkeit, diese Ausprägungen gezielt in den Fremdsprachenunterricht einzubeziehen.

\section{Bildungspolitische Vorgaben zur mündlichen Kommunikationsfähigkeit}

Die besondere Relevanz der mündlichen Sprachkompetenz kommt in den bildungspolitischen Dokumenten - vom Gemeinsamen europäischen Referenzrahmen für Sprachen (GeR) über die Bildungsstandards (Bista) bis zu den (Rahmen-) Lehrplänen der einzelnen Bundesländer - klar zum Ausdruck. Anschaulich belegen das die detaillierten Kompetenzerwartungen zu den verschiedenen Formen von Mündlichkeit. Neben Hörverstehen und Sprechen sowie ihren Teilkompetenzen finden auch weitere Aspekte Berücksichtigung: So führt der GeR spezielle Skalen zur Mündlichen Produktion (GeR 2001: 64-66) und zur Mündlichen Interaktion (GeR 2001: 79-85) an. Auch in den Bildungsstandards für die Allgemeine Hochschulreife „wird den funktionalen kommunikativen Kompetenzen, insb. der Mündlichkeit, sowie den interkulturellen Kompetenzen ein hoher Stellenwert eingeräumt“ (Bista 2012: 9-10). Dieser Prämisse folgend konkretisieren die länderspezifischen (Rahmen-)Lehrpläne - so auch für Russisch als zweite und dritte Fremdsprache - die Anforderungen für die einzelnen Kompetenzbereiche, die die Mündlichkeit konstituieren und zur angestrebten fremdsprachigen Handlungsfähigkeit beitragen.

Die inzwischen möglichen und geforderten Formen der mündlichen Leistungsfeststellung (vgl. mündliche Prüfungen als Teil der Prüfungen zum Mittleren Schulabschluss und Abiturprüfungen oder als Ersatz bzw. Teil einer Klassenarbeit in der Sekundarstufe I oder Klausur in der Sekundarstufe II) bekräftigen die besondere Relevanz der mündlichen Kommunikationsfähigkeit. Diese mündlichen Prüfungsformate tragen ihrerseits wieder dazu bei, die Mündlichkeit und insb. das Sprechen noch stärker in den Fokus zu rücken.

Die angeführten bildungspolitischen Vorgaben zur Entwicklung und Förderung von mündlicher Kommunikationsfähigkeit im Fremdsprachenunterricht vermitteln den Eindruck eines äußerst positiv geprägten Soll-Zustandes, der die

Anka Bergmann, Olga Caspers \& Wolfgang Stadler (Hg.)

Didaktik der slawischen Sprachen - Beiträge zum 1. Arbeitskreis in Berlin (12.-14.9.2016)

(C) 2018 innsbruck university press, ISBN 978-3-903187-11-5, DOI 10.15203/3187-11-5 
Ziele und Anforderungen in Hinblick auf ihre gesellschaftliche und persönliche Relevanz berücksichtigt und darüber hinaus einen zuverlässigen Handlungsrahmen für die Unterrichtspraxis bietet.

\section{Mündliche Kommunikationsfähigkeit in der schulischen Praxis}

Der Blick in die Klassenzimmer und ein von praktizierenden Lehrkräften konstatierter Ist-Zustand in Bezug auf die unterrichtliche Realität und das Kompetenzniveau der Lernenden weisen erfahrungsgemäß jedoch eher darauf hin, dass die Entwicklung und Förderung der mündlichen Kommunikationsfähigkeit und speziell des Sprechens nach wie vor eine große Herausforderung für Lernende und Lehrkräfte darstellen. Offensichtlich haben auch die Ergebnisse der DESIStudie (für den Englischunterricht) nichts von ihrer Aktualität verloren: „Anhand der Videodaten lässt sich nachweisen, dass die Lehrkraft im Durchschnitt doppelt so viel spricht wie alle Schüler zusammen. Auf Fragen der Lehrkraft erfolgt in der Hälfte aller Fälle innerhalb von drei Sekunden die Schülerantwort - wenn nicht, wird nur selten länger gewartet. Erfreulich ist, dass die Lehreräußerungen zu 84\%, die Schüleräußerungen zu 76\% auf Englisch erfolgen. Allerdings handelt es sich nur bei der Hälfte der Schüleräußerungen um frei formulierte englischsprachige Beiträge. Schülerfehler werden etwa in der Hälfte aller Fälle korrigiert, dann allerdings fast immer von der Lehrkraft, nicht von den Schülern selbst." (Klieme 2006: 6).

Im Rahmen einer Fortbildung (Juni 2016) bestätigten auch Russischlehrerinnen $(N=12)$ mit ihrem subjektiven Votum die Aktualität der Frage zum Sprechanteil von Lehrkräften und Lernenden im Unterricht. So schätzen zehn von ihnen den eigenen Sprechanteil mit $\geq 50 \%$ (s. Abb. 1) ein. Die individuelle Sprechzeit ihrer LernerInnen setzen sie zwischen fünf bis zehn Minuten oder auch weniger ${ }^{1}$ an (Abb. 2).

1 Die Befragten forderten den Zusatz „weniger“ ein, da ihrer Meinung nach SchülerInnen oft weniger als fünf Minuten Sprechzeit eingeräumt wird.

Anka Bergmann, Olga Caspers \& Wolfgang Stadler (Hg.)

Didaktik der slawischen Sprachen - Beiträge zum 1. Arbeitskreis in Berlin (12.-14.9.2016)

(C) 2018 innsbruck university press, ISBN 978-3-903187-11-5, DOI 10.15203/3187-11-5 


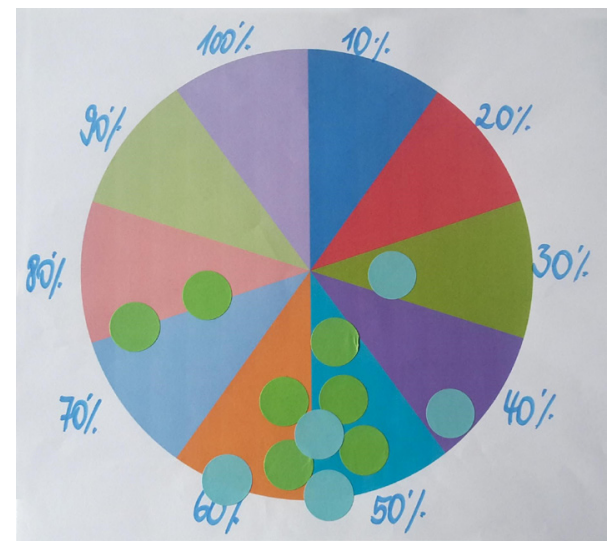

Abbildung 1: Durchschnittliche Sprechzeit als Lehrerin pro Unterrichtsstunde in \% $(N=12)$

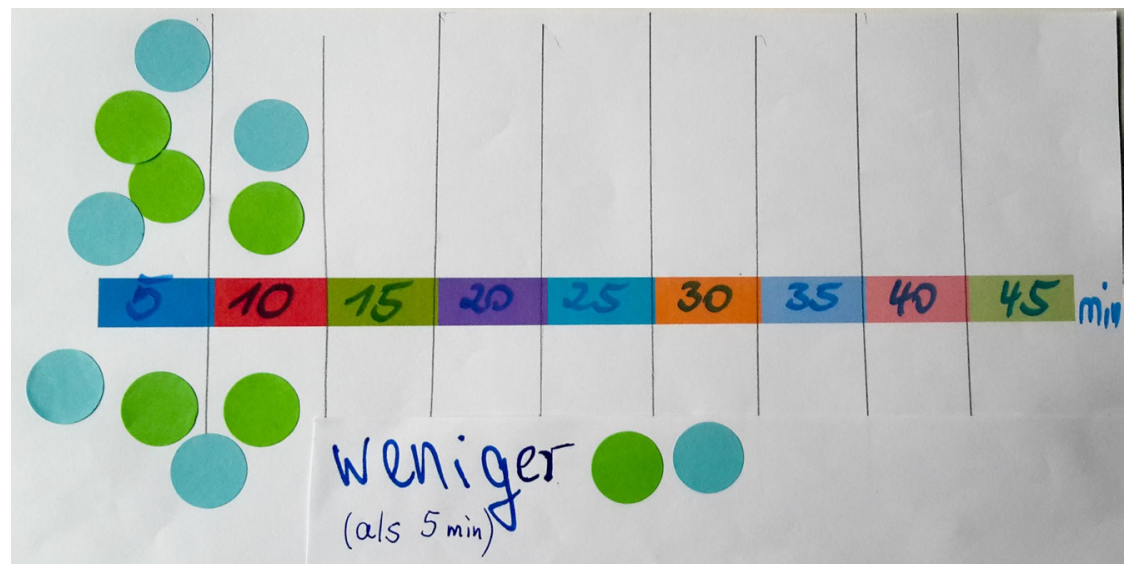

Abbildung 2: Durchschnittliche Sprechzeit der LernerInnen pro Unterrichtsstunde in Minuten

Wenn die mündlichen Kompetenzen, insb. das Sprechen, der Russischlernenden entwickelt und gefördert werden sollen, dann gilt zu beachten, dass „Sprechen nur entwickelt werden kann, wenn ausreichend Sprechzeit zur Verfügung steht“ (Behr \& Wapenhans 2014b: 139). Hürtgen (2017: 9) fordert „höhere Sprechzeiten für alle Schüler in jeder Stunde“ bzw. den „Redeanteil der Lehrkraft [...] 
zugunsten der Schüler zu reduzieren“ (ebd.: 11). Letztere müssen so oft wie möglich zu Wort kommen. Förderlich wirkt sich in diesem Zusammenhang aus, wenn es der Russischlehrkraft gelingt,

- eine offene und freundliche Atmosphäre im Russischunterricht zu schaffen, die das Kommunizieren-Wollen aller SchülerInnen befördert und ,Angst' vor dem Sprechen minimiert;

- vielfältige und motivierende Sprechanlässe und Gesprächssituationen anzubieten, die wirklich zum Sprechen anregen;

- geeignete Methoden und Verfahren einzusetzen, die ausreichend Gelegenheit zum mündlichen Üben und Anwenden bieten und zu längeren und zusammenhängenden Äußerungen herausfordern;

- regelmäßig Unterrichtsphasen einzuplanen, in denen alle gefordert sind, Russisch zu sprechen;

- den SchülerInnen ausreichend Zeit zum Überlegen und genügend Raum für komplexe Äußerungen zu geben;

- den ,stillen' SchülerInnen angemessene Hilfsangebote zu unterbreiten, aber auch die sprechfreudigen zu fördern;

- die nötige Geduld beim Warten auf Antworten der SchülerInnen aufzubringen;

- die russische Sprache konsequent als Unterrichtssprache (für Arbeitsanweisungen, Feedback, Unterrichtsorganisation u. ä.) einzusetzen;

- in mitteilungsbezogenen Phasen eine angemessene Fehlertoleranz zu zeigen und den SchülerInnen die Angst vor Fehlern zu nehmen;

- den LernerInnen die Selbstkorrektur zu ermöglichen.

Diese zehn praxisrelevanten Hinweise und die damit verbundenen didaktischen Entscheidungen müssen, wie die befragten Lehrerinnen einhellig bestätigten, von den Lehrkräften immer wieder reflektiert und auf allen Niveaustufen berücksichtigt werden. Gilt es doch, die mündliche Kommunikationsfähigkeit und speziell das Sprechen von Anfang an und im Folgenden kontinuierlich zu stärken. 


\section{Förderung der mündlichen Kommunikationsfähigkeit und die Anforderungen an die LehrerInnenbildung}

Es ist offensichtlich, dass die theoretischen und praktischen Anforderungen in Bezug auf die Förderung der mündlichen Kommunikationsfähigkeit nur zu bewältigen sind, wenn alle drei Phasen der LehrerInnenbildung, von der universitären Ausbildung über den Vorbereitungsdienst (Referendariat) bis zur Fort-/ Weiterbildung, diesem Thema die nötige Aufmerksamkeit widmen und die Ausprägung einer professionellen Handlungskompetenz, inkl. Wissen, Können und Einstellungen, in diesem Bereich unterstützen. Für die erste Phase der LehrerInnenbildung, d. h. konkret für die universitäre fachdidaktische sowie sprachpraktische Ausbildung, bedeutet das:

- die Studierenden für die Komplexität von Mündlichkeit zu sensibilisieren,

- Mündlichkeit mittels konkreter Lehr- und Lernszenarien erlebbar zu machen und

- verschiedene inhaltliche und fachdidaktische Ansatzpunkte zur Förderung von Mündlichkeit aufzuzeigen.

Diese Ansatzpunkte lassen sich modellhaft in vier verallgemeinernden Bereichen, die wechselseitig in Beziehung stehen, bündeln: (Lern-)Situation, Methoden und Verfahren, Differenzierung und Bewertung (s. Abb. 3).

Die gewählten Bezeichnungen ermöglichen eine relativ einfache Zuordnung der bereits aufgeführten praxisrelevanten Hinweise. So erfasst der Bereich „Lernsituation" viele der Aspekte, die in der Interaktion zwischen Lehrkraft und LernerInnen oder zwischen den Lernenden zum Tragen kommen: Sprechanlässe und -situationen, Sprechgelegenheiten und -phasen, Unterrichtsatmosphäre und -zeit etc. Der Bereich „Methoden und Verfahren“ bleibt nicht auf ein möglichst reichhaltiges Methodenrepertoire beschränkt, sondern schließt sowohl die Aufgabenkonstruktion mit entsprechenden Instruktionen und Operatoren als auch die russischsprachige Unterrichtskommunikation und die bewusste Wahl der Sozialformen ein. „Differenzierung“ wird erfahrbar durch inhaltliche und sprachliche Unterstützungs- und Fördermaßnahmen, die insb. den Bedürfnissen heterogener

Anka Bergmann, Olga Caspers \& Wolfgang Stadler (Hg.)

Didaktik der slawischen Sprachen - Beiträge zum 1. Arbeitskreis in Berlin (12.-14.9.2016)

(C) 2018 innsbruck university press, ISBN 978-3-903187-11-5, DOI 10.15203/3187-11-5 


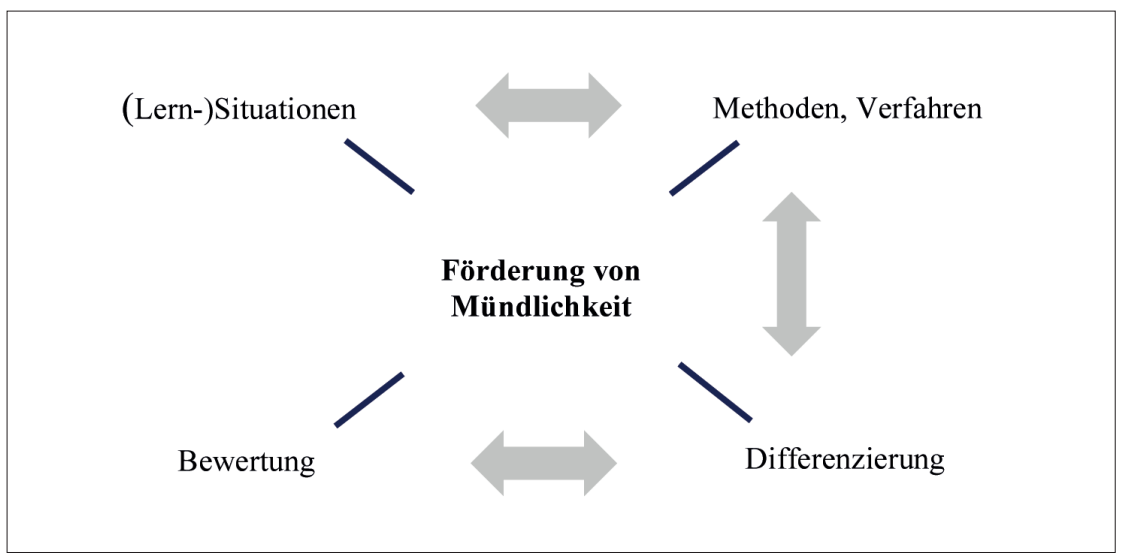

Abbildung 3: Fachdidaktische Ansatzpunkte zur Förderung von Mündlichkeit

Lerngruppen gerecht werden. In den Bereich „Bewertung“ fallen die möglichen Formen und Maßstäbe bei der Erfassung und Einschätzung bzw. Beurteilung mündlicher Leistungen.

Die genannten vier Bereiche werden im Bachelor- und Masterstudium für Lehramtsstudierende in den verschiedenen Lehrveranstaltungsformaten der Fachdidaktik in unterschiedlichem Maße thematisiert und darüber hinaus in der Sprachpraxis individuell erlebt. Überaus wichtig erscheint es z. B., dass Lehramtsstudierende in der universitären Ausbildungsphase das Prinzip der Unterrichtskommunikation „so viel Russisch wie möglich und so wenig Deutsch wie nötig“ selbst erfahren und die existierenden Hilfestellungen in Form von Listen mit Arbeitsanweisungen und Unterrichtswendungen in russischer Sprache (vgl. die aktuellen Russischlehrwerke und einzelne Publikationen, z. B. Behr \& Wapenhans 2014a: 12, Wapenhans 2013a: 13, 2013b: 13) kennenlernen.

Methoden und Verfahren, die im eigenen Sprachlernprozess erlebt, ausprobiert und als nutzbringend und effektiv eingeschätzt wurden, kommen in der beruflichen Praxis eher zum Einsatz als solche, die nur in der Fachliteratur rezipiert wurden. Diese Annahme fußt auf eigenen Beobachtungen im Schulpraktikum und zahlreichen Gesprächen mit Studierenden während der Vorbereitung, Durchführung und Auswertung ihres Praktikums sowie mit den im Referendari- 
at tätigen Lehrkräften und ehemaligen Studierenden. Eine empirische Erhebung dazu steht aus, soll aber in nächster Zeit realisiert werden.

Demzufolge erscheint es als besonders wichtig, möglichst viele Methoden und Verfahren sinnvoll in die fachdidaktischen und sprachpraktischen Lehrveranstaltungen zu integrieren und sie, vorzuleben'. Geissler (1985, zitiert nach Wahl 2013: 66) bezeichnet dies als „pädagogischen Doppeldecker“. Eine sich anschließende gemeinsame Analyse und Reflexion kann zur Entwicklung eines flexiblen Methodenrepertoires der zukünftigen Russischlehrkräfte beitragen, das sie weiterführend in ein fächerübergreifendes Methodencurriculum (vgl. Ziener \& Kessler 2012: 200ff.) einbringen.

\section{Methodenrepertoire zur Förderung der mündlichen Kommunikationsfähigkeit}

Es existiert eine Reihe von Publikationen, die verschiedene Methoden und Verfahren für die Förderung mündlicher Kommunikationsfähigkeit im Englisch-, Französisch- und Spanischunterricht empfehlen (vgl. Grieser-Kindel, Möller \& Henseler 2006, 2009, Klein \& Schönfeld 2012, Neveling, Hoyer \& Zausch 2012, Ritter et al. 2011, Sobel 2014, Steveker 2014, Ziener \& Kessler 2013, Zerck 2014). Unter den vorgestellten Methoden sind nicht wenige, die insb. das Sprechen unterstützen sowie die individuelle Sprechzeit des/der einzelnen Schülers/Schülerin erhöhen, z. B. Kugellager, Think-Pair-Share, Vier-Ecken-Gespräch, Gruppenpuzzle. Auch wenn es bisher keine aufbereiteten Methodensammlungen speziell für den Russischunterricht gibt, ist davon auszugehen, dass Russischlehrkräfte bekannte Methoden aus dem Unterricht anderer Fremdsprachen zur SchülerInnenaktivierung und zum kooperativen Lernen auf den verschiedenen Niveaustufen einsetzen. Die notwendige inhaltliche und sprachliche Anpassung, inkl. der russischsprachigen Aufgabenstellungen, muss in der Regel individuell geleistet werden, wenn nicht auf einzelne Veröffentlichungen der Zeitschrift PRAXIS Fremdsprachenunterricht (Russisch) zurückgegriffen werden kann, wie z. B. auf die praxiserprobten Empfehlungen zu folgenden Methoden: Gruppenpuzzle (Bayer 2014), Placemat (Fitzer 2014), Talkshow (Voloshyna 2013).

Anka Bergmann, Olga Caspers \& Wolfgang Stadler (Hg.)

Didaktik der slawischen Sprachen - Beiträge zum 1. Arbeitskreis in Berlin (12.-14.9.2016)

(C) 2018 innsbruck university press, ISBN 978-3-903187-11-5, DOI 10.15203/3187-11-5 
So wie für alle zukünftigen Fremdsprachenlehrenden ist es auch für angehende Russischlehrkräfte von besonderer Wichtigkeit, eine Vielzahl an Methoden zu kennen, diese in Bezug auf ihr Potential im Russischunterricht zu analysieren und dann bedarfsgerecht zu adaptieren. Von fachdidaktischem Interesse sind aus diesem Grund die Antworten auf die Fragen, welche Methoden und Verfahren zur Förderung der mündlichen Kommunikationsfähigkeit den Bachelor- und Masterstudierenden bereits bekannt sind, um einerseits ihr Methodenbewusstsein zu schärfen sowie ihre Selbstreflexion anzuregen und andererseits gezielt weitere Methoden in die folgenden Lehrveranstaltungen einzubinden.

\section{Befragung zum Methodenrepertoire}

Im Sommersemester 2016 wurden zwölf Studierende einer Bachelorgruppe und sechs Studierende einer Mastergruppe im Rahmen ihrer jeweiligen fachdidaktischen Lehrveranstaltungen am Institut für Slawistik der Humboldt-Universität zu Berlin aufgefordert, eine Liste mit insgesamt 32 verschiedenen Methoden zur Förderung des Sprechens im Russischunterricht zu bearbeiten und mittels Ankreuzen zu vermerken, welche sie kennen, welche sie als LernerInnen erlebt und welche sie im Schulpraktikum selbst ausprobiert haben. Die konkreten Methoden wurden im Wesentlichen den oben genannten Methodensammlungen entnommen und in alphabetischer Reihenfolge aufgeführt (s. Tab. 1).

Tabelle 1: Alphabetische Auflistung der abgefragten Methoden zur Förderung des Sprechens

\begin{tabular}{|cl|}
\hline 1. & Aquarium \\
\hline 2. & Auswischtechnik/Ausgewischt \\
\hline 3. & Blitzlicht \\
\hline 4. & Denken-Austauschen-Besprechen \\
\hline 5. & Domino \\
\hline 6. & Drei-Schritt-Interview \\
\hline 7. & Ein-Minuten-Vortrag/Kurzpräsentation \\
\hline 8. & Einer bleibt - drei gehen \\
\hline
\end{tabular}




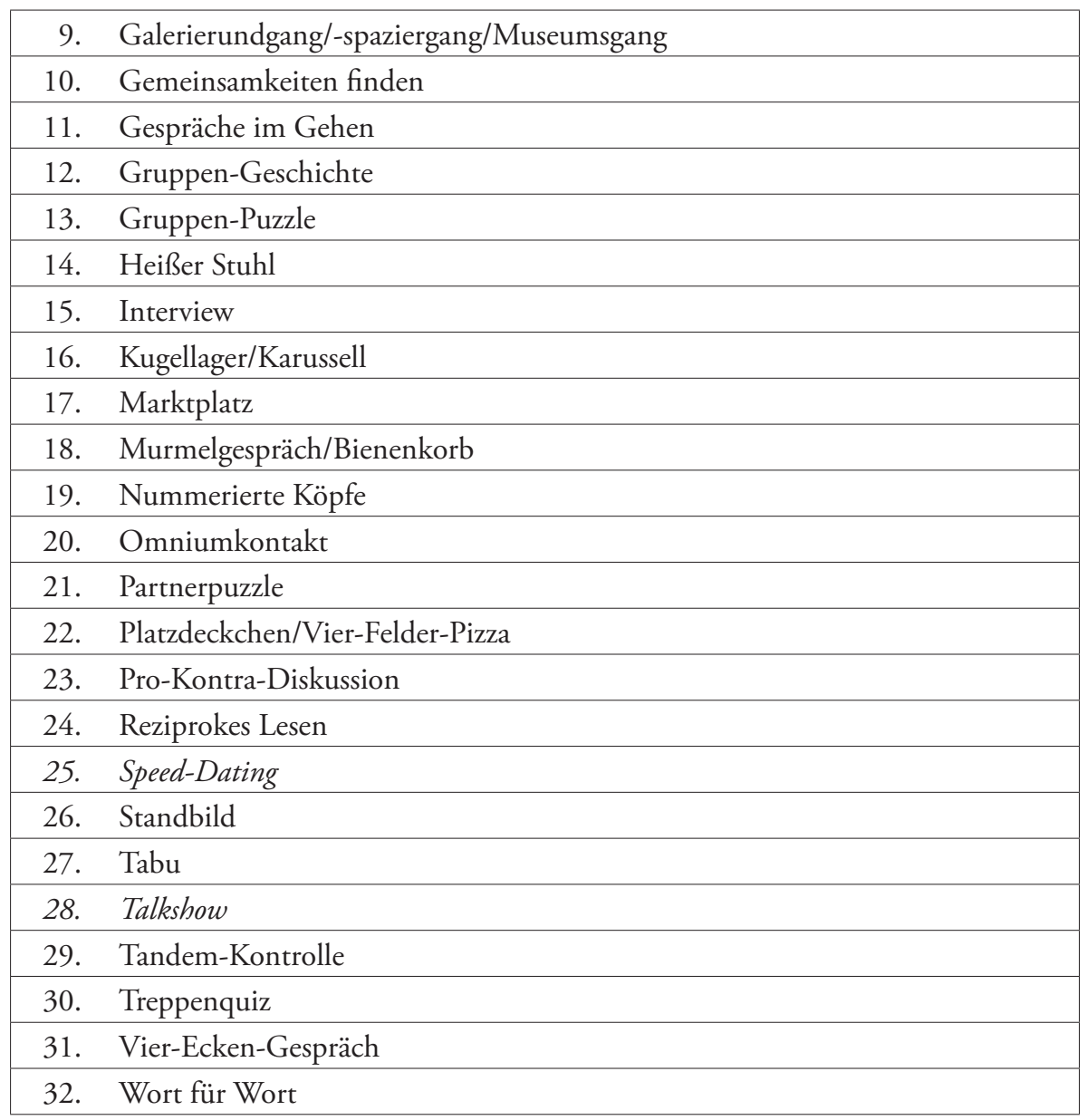

Die Auswertung der Umfrage ergab, dass die zwölf befragten Bachelor-Studierenden im Durschnitt ca. $13(12,8)$ der 32 aufgelisteten Methoden kannten und bereits mind. acht $(8,4)$ Methoden selbst erlebt haben (s. Abb. 4). ${ }^{2}$

2 Der Nullwert für bereits erlebte Methoden des unter Nr. 4 aufgelisteten Fragebogens erklärt sich möglicherweise daraus, dass ein Studierender zu spät kam und ihm dann die Zeit zum bewussten Ankreuzen fehlte. 


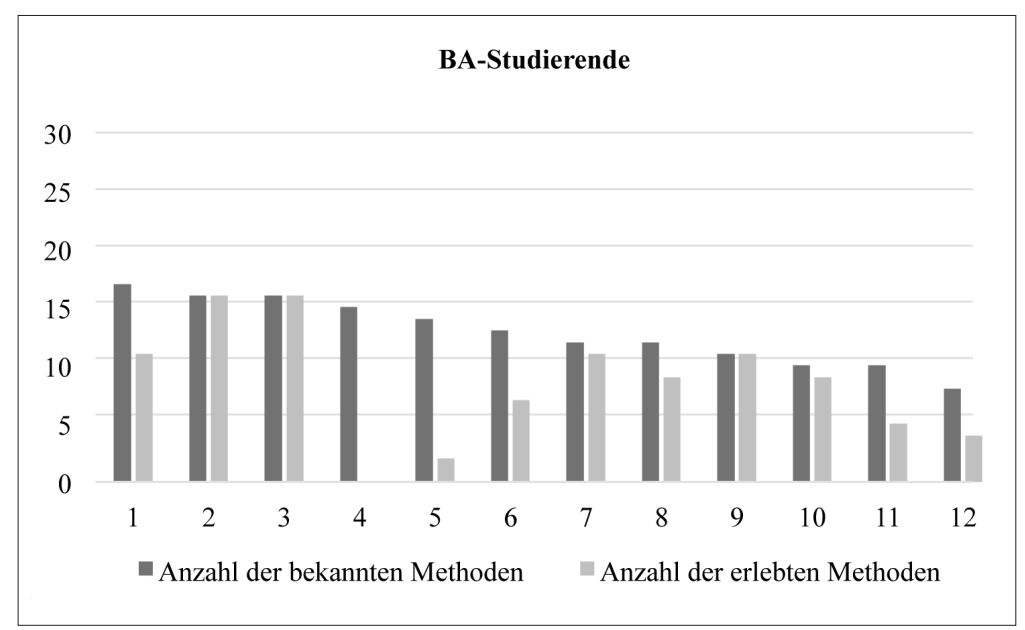

Abbildung 4: Anzahl der bekannten und erlebten Methoden von Bachelor-Studierenden $(N=12)$

Die Werte der Masterstudierenden (s. Abb. 5) belegen, dass durchschnittlich bereits $17(17,3)$ der 32 Methoden bekannt sind, im Falle von vier der befragten Studierenden sogar mehr als 20. Die Anzahl der selbst erlebten Methoden unterscheidet sich jedoch nicht wesentlich von jener, die von den Bachelorstudierenden angegeben wurde: 9,7 im Vergleich zu 8,4. Fünf der befragten Masterstudierenden hatten bereits das Schulpraktikum absolviert, ihnen können damit mit großer Sicherheit die Angaben zu den ausprobierten Methoden zugeordnet werden (s. Abb. 5).

Der Vergleich der Werte in Bezug auf bekannte und erlebte Methoden legt die Schlussfolgerung nahe, dass in der Bachelorphase bei ca. der Hälfte der Studierenden die Methodenkenntnis auf den selbst erlebten Methoden beruht. Bei den Masterstudierenden ist ein theoretischer Vorsprung festzustellen, da sie angeben, mehr Methoden zu kennen als selbst erlebt zu haben.

Interessanterweise entwickelte sich in beiden befragten Gruppen nach Abgabe der Fragebögen eine lebhafte Diskussion zu den Methoden und den mit ihnen verbundenen Inhalten. So zeigte sich, dass Studierende mit einem zweiten Fach im Bereich der Fremdsprachen oder Geschichte versierter mit den Methodenbe- 


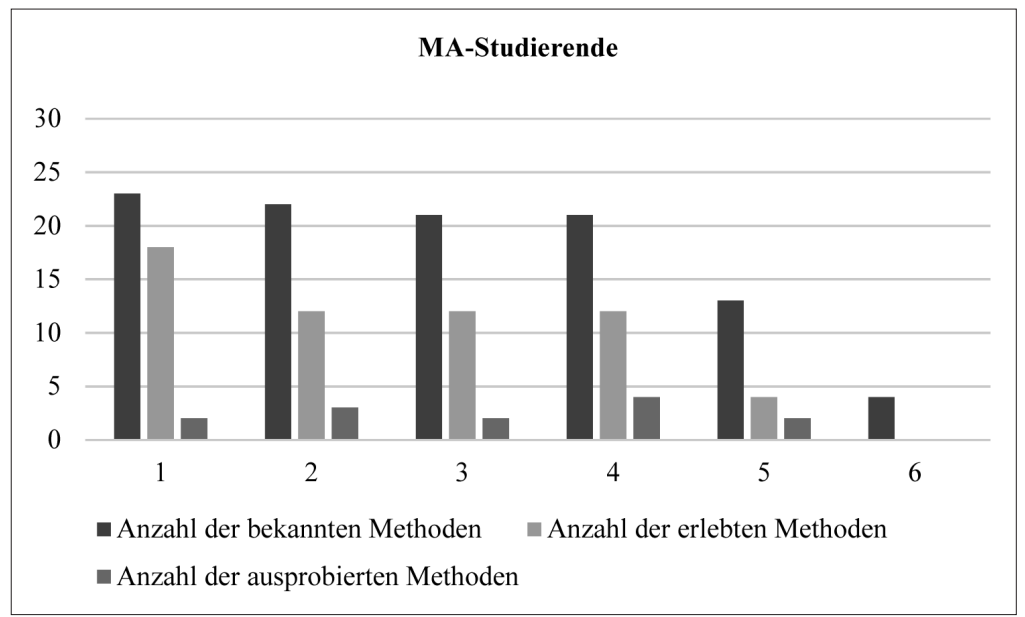

Abbildung 5: Anzahl der bekannten, erlebten und ausprobierten Methoden von Master-Studierenden $(N=6)$

zeichnungen umgehen konnten und die Methodenliste durch eigene Vorschläge wie Bingo und Laufgespräch bzw. Talking while walking (Gespräche im Gehen) ergänzten. Auch der Rückgriff auf eigene Lernerfahrungen in den sprachpraktischen Lehrveranstaltungen für Russisch oder Deutsch als Fremdsprache erwies sich als sehr nützlich. Einige Studierende gaben im Gespräch außerdem zu verstehen, dass sie offensichtlich doch mehr Methoden kennen, ohne dass ihnen die genauen Benennungen dafür zur Verfügung stehen. Offen wurde der Wunsch geäußert, die Lehrveranstaltungen in der Fachdidaktik, der Sprachpraxis, aber auch den Fachwissenschaften noch stärker als ,Muster ' für Methodenvielfalt zu gestalten und die zur Anwendung kommenden Aktivitäten nicht auf Partner- und Gruppenarbeit zu beschränken. Die Studierenden wollen nicht nur über Methoden ,reden', sondern sie im praktischen Einsatz sehen bzw. selbst Lernszenarien entwickeln und anschließend besprechen.

Als Hilfsmittel für die weitere Arbeit am eigenen Methodenrepertoire erhielten die Studierenden die Liste mit den 32 Methoden (s. Tab. 1), die für jede Methode um konkrete Literaturhinweise zum Nachlesen ergänzt wurde. 


\section{Fazit}

Die Bestandsaufnahme zum Soll- und Ist-Stand bezüglich der mündlichen Kommunikationsfähigkeit im Fremdsprachenunterricht hat gezeigt, welchen Anforderungen die 1. Phase der LehrerInnenbildung gerecht werden sollte, um die zukünftigen Russischlehrkräfte in diesem Bereich optimal auf die Unterrichtspraxis vorzubereiten. Neben der Sensibilisierung für die Komplexität der mündlichen Kommunikationsfähigkeit kommt es darauf an, die Ansatzpunkte zur Förderung von Mündlichkeit bewusst zu machen und die entsprechenden Komponenten zu thematisieren. Einen wichtigen Bereich stellen dabei Methoden und Verfahren dar. Diese gilt es nicht nur auf der Wissensebene in Form von sog. Methodenlisten zu vermitteln, sondern auch bewusst in die Ausbildung zu integrieren, um sie dann gemeinsam mit den Studierenden zu analysieren und zu reflektieren. Methodenvielfalt sollte dabei ein Anspruch aller an der Ausbildung von Russischlehrkräften beteiligten AkteurInnen sein - von der Sprachpraxis über die philologischen Fachwissenschaften bis zur Fachdidaktik.

\section{Literaturverzeichnis}

Bayer, M. (2012). Kak v Rossii vstrečajut Novyj god. Ein Gruppenpuzzle zum Ausklang des Jahres. PRAXIS Fremdsprachenunterricht Russisch, 6, 6-8.

Behr, U. \& Wapenhans, H. (2014a). Was soll ich tun? Arbeitsanweisungen verstehen und umsetzen. PRAXIS Fremdsprachenunterricht Russisch, 1, 12.

Behr, U. \& Wapenhans, H. (2014b). Sprechen als komplexe Sprachaktivität im Russischunterricht. In A. Bergmann (Hrsg.), Fachdidaktik Russisch. Eine Einführung (134-145). Tübingen: Narr.

Bista=Bildungsstandards für die fortgeführte Fremdsprache (Englisch / Französisch) für die Allgemeine Hochschulreife (2012). Verfügbar unter: https://tinyurl.com/ybkwzcqo [02.12.2017].

Fitzer, T. (2014). Kak my proveli letnie kanikuli. Die erste Stunde nach den Ferien. PRAXIS Fremdsprachenunterricht Russisch, 4, 10-11.

GeR=Trim, J., North, B. \& Coste, D. (Hrsg.) (2001). Gemeinsamer europäischer Referenzrahmen für Sprachen: Lernen, lehren, beurteilen. Berlin: Langenscheidt. 
Grieser-Kindel, C., Möller, S. \& Henseler, R. (2006). Method Guide. Schüleraktivierende Methoden für den Englischunterricht in den Klassen 5-10. Paderborn: Schöningh.

Grieser-Kindel, C., Henseler, R. \& Möller, S. (2009). Method Guide. Methoden für einen kooperativen und individualisierenden Englischunterricht in den Klassen 5-12. Paderborn: Schöningh.

Hürtgen, A. (2017). Fremdsprachenunterricht: Mündliche Kommunikation trainieren: Sprechanteile erhöhen, Kommunikationsanlässe schaffen, Redemittel bereitstellen. Berlin: Cornelsen.

Klein, C. \& Schönfeld, J. (2012). Methodenkiste für einen kommunikativen Fremdsprachenunterricht. Lehrerselbstverlag (Fremdsprachen Lehren und Lernen).

Klieme, E. (2006). Zusammenfassung zentraler Ergebnisse der DESI-Studie. Verfügbar unter: https://tinyurl.com/yardxyn5 [02.12.2017].

Kurtz, J. (2014). Transformative Mündlichkeit: Eine Frage des Taktes. In E. Burwitz-Melzer, F. G. Königs \& Riemer, C. (Hrsg.), Grieser-Kindel, C., Henseler, R. \& Möller, Perspektiven der Mündlichkeit. Arbeitspapiere der 34. Frühjahrskonferenz zur Erforschung des Fremdsprachenunterrichts. (= Giessener Beiträge zur Fremdsprachendidaktik) (117-126). Tübingen: Narr.

Neveling, C., Hoyer, B. \& Zausch, A. (2012). Unterrichtsverfahren zur Förderung der Sprechkompetenzen. Französisch heute, 3, 107-115.

Ritter, M., Rogge, M., Winz, P. \& Wirtz-Kaltenberg, P. (Hrsg.) (2011). Let's talk! Eine Handreichung für die mündlichen Prüfungen in den Klassenstufen 5-12. Berlin: Cornelsen.

Sobel, M. (2014). Das schnelle Methoden 1x1 Französisch mit Arbeitsmaterialien. Berlin: Cornelsen.

Steveker, W. (2014). Das schnelle Methoden 1x1 Spanisch mit Arbeitsmaterialien. Berlin: Cornelsen.

Voloshyna, T. (2013). Graffiti - iskusstvo ili vandalizm. Tok-šou „Duel' mnenij“. PRAXIS Fremdsprachenunterricht Russisch, 6, 8-11.

Wahl, D. (2013). Lernumgebungen erfolgreich gestalten. Vom trägen Wissen zum kompetenten Handeln. Bad Heilbrunn: Klinkhardt.

Wapenhans, Н. (2013а). Браво! Молодцы, ребята! Unterrichtssprache unter dem Aspekt der Förderung (1). PRAXIS Fremdsprachenunterricht Russisch, 3, 13.

Wapenhans, Н. (2013b). Браво! Молодцы, ребята! Unterrichtssprache unter dem Aspekt der Förderung (2). PRAXIS Fremdsprachenunterricht Russisch, 5, 13.

Wolff, D. (2000). Sprachproduktion als Planung: ein Beitrag zur Psychologie des Sprechens. Der Fremdsprachliche Unterricht Englisch, 5, 11-16.

Zerck, K. (2014). Das schnelle Methoden $1 \times 1$ Englisch mit Arbeitsmaterialien. Berlin: Cornelsen.

Ziener, G. \& Kessler M. (2013). Kompetenzorientiert unterrichten - mit Methode. Methoden entdecken, verändern, erfinden. Seelze: Friedrich Verlag.

Anka Bergmann, Olga Caspers \& Wolfgang Stadler (Hg.)

Didaktik der slawischen Sprachen - Beiträge zum 1. Arbeitskreis in Berlin (12.-14.9.2016)

(C) 2018 innsbruck university press, ISBN 978-3-903187-11-5, DOI 10.15203/3187-11-5 
